
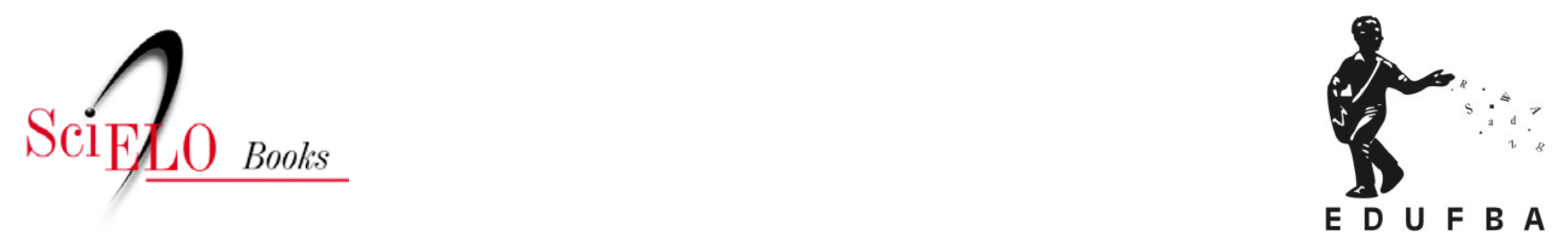

\title{
Saúde mental entre presidiários na cidade do Salvador, Bahia, Brasil
}

\author{
Antônio Carlos Cruz Freire \\ Milena Pereira Pondé \\ Milena Siqueira Santos Mendonça
}

\section{SciELO Books / SciELO Livros / SciELO Libros}

FREIRE, A.C.C., PENDÉ, M.P. and MENDONÇA, M.S.S. Saúde mental entre presidiários na cidade do Salvador, Bahia, Brasil. In: COELHO, M.T.Á.D., and CARVALHO FILHO, M.J., orgs. Prisões numa abordagem interdisciplinar [online]. Salvador: EDUFBA, 2012, pp. 121-130. ISBN 978-85232-1735-8. Available from: doi: 10.7476/9788523217358.008. Also available in ePUB from: http://books.scielo.org/id/7mkg8/epub/coelho-9788523217358.epub.

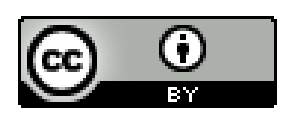

All the contents of this work, except where otherwise noted, is licensed under a Creative Commons Attribution $\underline{4.0 \text { International license. }}$

Todo o conteúdo deste trabalho, exceto quando houver ressalva, é publicado sob a licença Creative Commons Atribição 4.0.

Todo el contenido de esta obra, excepto donde se indique lo contrario, está bajo licencia de la licencia Creative Commons Reconocimento 4.0. 


\title{
Saúde mental entre presidiários na cidade do Salvador, Bahia, Brasil ${ }^{1}$
}

\author{
Antônio Carlos Cruz Freire \\ Milena Pereira Pondé \\ Milena Siqueira Santos Mendonça
}

\section{Introdução}

O número de pessoas vivendo em um sistema prisional em todo mundo ultrapassa nove milhões, porém o número de indivíduos acometidos por transtorno psiquiátrico grave nestas instituições é impreciso. (FAZEL; DANESH, 2002) Estudos realizados em diferentes países estimam que os transtornos mentais graves sejam 5 a 10 vezes mais prevalentes entre presos do que na população geral. (BRUGHA et al., 2005; FALISSARD et al., 2006) Transtornos graves de personalidade e transtornos relacionados ao uso de substâncias psicoativas são comuns nesta população. Essas enfermidades têm caráter crônico e não contam com abordagens terapêuticas fáceis. (MORRIS, 2002) Depressão, transtornos ansiosos e transtornos relacionados ao sono são também frequentes, porém, muitas vezes ocorrem associados aos transtornos de personalidade ou uso de substâncias psicoativas. ${ }^{5}$ (WEXLER, 2003)

Os presos têm o mesmo direito de assistência psiquiátrica que o restante da população, contudo poucos pacientes recebem assistência

1 Este trabalho foi realizado com o apoio da FAPESB através do Edital de Segurança Pública (FAPESB/SECTI/SSP). Termo de Outorga ET-06/2005. 
psiquiátrica de maneira adequada durante o período de aprisionamento. Isto representa, em primeira instância, a perda da possibilidade de tratar indivíduos que, habitualmente, não entram em contato com serviços de saúde mental. (SMITH et al., 2003; BIRMINGHAM et al., 1998; LANGEVELD; MELHUS, 2004)

Um estudo australiano examinou se haveria uma maior prevalência de doenças psiquiátricas entre presos quando comparados com a população geral, após realização de ajustes de variáveis demográficas. Utilizando o Composite International Diagnostic Interview (CIDI) como instrumento diagnóstico, estimou-se que a prevalência de transtornos psiquiátricos em prisioneiros foi de $80 \%$, enquanto na comunidade a prevalência foi de 31\%. (BUTLER et al., 2006) Principalmente sintomas de psicose (OR $=11,8,95 \%$ CI 7,5-18,7), transtornos relacionados ao uso de substâncias psicoativas (OR $=11,4$, 95\% CI 9,7-13,6) e transtornos de personalidade (OR = 8,6, 95\% CI 7,2-10,3) (BUTLER et al., 2006).

Uma extensa revisão sistemática da literatura estimou que 3,7\% da população carcerária apresentava um transtorno psicótico, 10\% depressão e $42 \%$ transtorno de personalidade ${ }^{1}$. Na França a prevalência de transtornos mentais em presos do sexo masculino foi estimada através de uma entrevista clínica semi-estruturada (MINI Plus V. 5.0) aplicada por médico e posteriormente entrevista não estruturada com psiquiatra. As seguintes prevalências foram encontradas: depressão 23\%, transtornos ansiosos 13\%, dependência de álcool 8,7\% e drogas 8,.9\%, transtornos psicóticos 13,4\% (FALISSARD et al., 2003). A prevalência de doença mental entre presos não condenados do sexo masculino na Inglaterra e País de Gales foi estimada através de entrevista semi-estruturada, com base nos critérios diagnósticos CID 10: depressão 9,5\%, dependência de álcool e drogas 38\%, transtornos psicóticos 4,8\% e transtorno de personalidade $11,2 \%{ }^{10}$. Um estudo realizado no Irã evidenciou, através da aplicação de escalas semi-estruturadas (SCID CV e PCL SV), aplicadas por médicos residentes em psiquiatria, as seguintes prevalências: depressão 29,1\%; psicose 3,1\%; dependência de opióide ao longo da vida 72,7\%; álcool 8,8\% e psicopatia 25,5\% (ASSADI et al., 2006).

Um estudo australiano examinou se haveria uma maior prevalência de doenças psiquiátricas entre presos quando comparados com a 
população geral, após realização de ajustes de variáveis demográficas. Utilizando o Composite International Diagnostic Interview (CIDI) como instrumento diagnóstico, estimou-se que a prevalência de transtornos psiquiátricos em prisioneiros foi de $80 \%$, enquanto na comunidade a prevalência foi de 31\%. (BUTLER et al., 2006) Principalmente sintomas de psicose (OR $=11,8,95 \%$ CI 7,5-18,7), transtornos relacionados ao uso de substâncias psicoativas (OR $=11,4$, 95\% CI 9,7-13,6) e transtornos de personalidade (OR $=8,6,95 \%$ CI 7,2-10,3). (BUTLER et al., 2006)

Uma extensa revisão sistemática da literatura estimou que 3,7\% da população carcerária apresentava um transtorno psicótico, 10\% depressão e $42 \%$ transtorno de personalidade ${ }^{1}$. Na França a prevalência de transtornos mentais em presos do sexo masculino foi estimada através de uma entrevista clínica semi-estruturada (MINI Plus V. 5.0) aplicada por médico e posteriormente entrevista não estruturada com psiquiatra. As seguintes prevalências foram encontradas: depressão 23\%, transtornos ansiosos 13\%, dependência de álcool 8,7\% e drogas $8,9 \%$, transtornos psicóticos 13,4\%. (FALISSARD et al., 2003) A prevalência de doença mental entre presos não condenados do sexo masculino na Inglaterra e País de Gales foi estimada através de entrevista semi-estruturada, com base nos critérios diagnósticos CID 10: depressão 9,5\%, dependência de álcool e drogas 38\%, transtornos psicóticos 4,8\% e transtorno de personalidade 11,2\%. ${ }^{10}$ Um estudo realizado no Irã evidenciou, através da aplicação de escalas semi-estruturadas (SCID CV e PCL SV), aplicadas por médicos residentes em psiquiatria, as seguintes prevalências: depressão 29,1\%; psicose 3,1\%; dependência de opióide ao longo da vida 72,7\%; álcool 8,8\% e psicopatia 25,5\%. (ASSADI et al., 2006)

Os estudos citados sugerem que a população carcerária necessita de cuidados psiquiátricos para transtornos mentais graves, indicando também que talvez a melhor atenção a esses doentes possa reduzir as taxas de criminalidade. A saúde mental dos presos no Brasil não foi estudada anteriormente. Nós realizamos um estudo de prevalência de transtornos mentais em presos condenados no Estado da Bahia com o objetivo de conhecer o perfil psiquiátrico dos presos deste Estado, através de inquérito semiestruturado baseado nos critérios diagnósticos do DSM IV, adotando um estudo de corte transversal, feito com uma 
amostra de detentos do sexo masculino de duas instituições carcerárias da cidade do Salvador, Bahia, Brasil. A primeira instituição foi um Complexo Penitenciário para cumprimento de pena em regime fechado. A segunda, uma Colônia Penal, em regime semi-aberto. A coleta de dados foi realizada entre os meses de junho e novembro de 2006. O instrumento utilizado foi a tradução para o português do MINI plus (AMORIM, 2000), que fornece o diagnóstico psiquiátrico pelo DSM-IV (manual diagnóstico e estatístico dos transtornos mentais, da Associação Americana de Psiquiatria) e pela Classificação Internacional das Doenças (CID-10), da Organização Mundial de Saúde.

\section{O complexo penitenciário}

Esta instituição abrigava 2343 condenados para cumprimento de pena em regime fechado. O presídio estava dividido em cinco pavilhões, que por seu turno se subdividiam em galerias, que abrigavam as respectivas celas. Os presos a serem entrevistados foram sorteados aleatoriamente através da lista dos detentos fornecida pela direção do presídio. Foram excluídos aqueles que não puderam ser localizados através do sorteio no pátio porque haviam sido transferidos para outro presídio ou outro regime prisional, porque haviam fugido ou porque já haviam sido postos em liberdade. Foram excluídos também os presos que se recusaram a participar da pesquisa, que foram em número inferior a 10.

Foram entrevistados 290 presos, a idade variou de 20 a 63 anos, sendo a média de 33 anos. A depressão ocorreu entre 8 e 17 anos para $12(4,1 \%)$ pessoas, entre 18 e 30 anos para 33 (11,4\%), entre 31 e 54 anos para $6(2 \%)$. A maioria das pessoas teve apenas um episódio depressivo durante a vida 31 (10,7\%), enquanto 20 (6,9\%) indivíduos tiveram entre 2 a 20 episódios depressivos ao longo da vida. O transtorno afetivo bipolar foi o diagnóstico de 19 (6,6\%) detentos, sendo que $4(1,3 \%)$ indivíduos tiveram seus sintomas de humor desencadeados pelo uso de substâncias psicoativas ou medicamentos. O transtorno de ansiedade generalizada foi detectado em 12 (4,1\%) detentos, quadros psicóticos foram detectados em 4 (1,4\%), personalidade antissocial em 78 (26,9\%) e personalidade borderline em 57 (19,7\%) dos internos. O uso abusivo ou dependência de álcool durante a vida foi positivo para 
177 (61\%) presos. No regime fechado as substâncias mais utilizadas e referidas como relacionadas à dependência ao longo da vida foram a maconha 43 (14,8\%), cocaína ou crack 35 (12\%) e os inalantes $3(0,9 \%)$ ou benzodiazepínicos. Com relação à dependência atual de substâncias, 12 (4\%) presos referiam problemas nos últimos 12 meses com maconha. O tipo de substância de abuso atual foi a maconha para 19 $(6,5 \%)$ e estimulante (cocaína ou crack) para 2 (0,6\%). A presença de transtorno de déficit de atenção e hiperatividade (TDAH) na infância foi positiva para 30 internos (10,3\%), enquanto a presença dos sintomas na vida adulta persistiu em 12 presos $(4,1 \%)$.

\section{A colônia penal}

Abrigava 354 condenados cumprindo pena em regime semi-aberto. Neste regime, com autorização judicial, o preso tem o direito de sair em datas festivas, bem como trabalhar fora do presídio e retornar diariamente para a Colônia após a jornada de trabalho. O cumprimento da pena em quaisquer dos regimes é fixado em sentença condenatória, que considera o tempo da pena e o grau de periculosidade do condenado. Todos os condenados terão direito à progressão de regime conforme critério de elegibilidade: do regime fechado para o semiaberto; do semiaberto para o aberto. O condenado que cumprir os requisitos previstos em lei - o cumprimento de fração da pena e bom comportamento prisional - poderá requerer a progressão do seu regime.

Todos os presos que se encontravam no local durante o dia foram entrevistados. Foram excluídos aqueles que não foram encontrados porque estavam trabalhando ou haviam fugido, ou haviam sido transferidos por progressão ou regressão de regime, ou para o hospital de custódia, por apresentarem transtorno mental grave. Foram excluídos também detentos que não concordaram em participar da pesquisa, que foram em número inferior a 10.

Foram entrevistados 207 presos, a idade variou de 19 a 65 anos, sendo a média 29,5 anos, não havendo portanto diferença significativa entre as médias de idade entre os presos dos dois regimes. A depressão ocorreu entre 7 e 17 anos para 12 (5,7\%) pessoas, entre 18 e 30 anos para $20(9,6 \%)$, entre 31 e 65 anos para $7(3,5 \%)$. A maioria das pessoas teve apenas um episódio depressivo durante a vida 21 (10\%), enquanto 
$18(8,7 \%)$ indivíduos tiveram entre 2 a 30 episódios depressivos ao longo da vida.

O transtorno afetivo bipolar foi o diagnóstico de 32 (15,5\%) detentos, sendo que $11(5,3 \%)$ indivíduos tiveram seus sintomas de humor desencadeados pelo uso de substâncias psicoativas ou medicamentos. O transtorno de ansiedade generalizada foi detectado em 15 (7,2\%) detentos, quadros psicóticos foram detectados em 26 (12,6\%), personalidade anti-social em $50(24,2 \%)$ e personalidade borderline em 72 (35\%) dos internos. O uso abusivo ou dependência de álcool durante a vida foi positivo para $140(67,7 \%)$ presos. No regime semiaberto o tipo de substância mais utilizada e causadora de dependência ao longo da vida foi maconha 42 (20\%), cocaína ou crack 24 (11,5\%), seguido de inalantes 1 (0,5\%). Com relação à dependência atual de substâncias, 20 $(9,5 \%)$ presos referiam problemas nos últimos 12 meses com maconha e três internos $(1,5 \%)$ referiam dependência atual a cocaína ou crack. O tipo de substância de abuso atual mais comum foi a maconha para 20 (9,5\%) indivíduos, seguido de estimulante (cocaína ou crack) para

1263 (1,5\%). A Presença de Transtorno de Déficit de Atenção e Hiperatividade (TDAH) na infância foi positiva para 46 internos $(22,2 \%)$, enquanto à presença dos sintomas na vida adulta, persistiram em 11 presos $(5,3 \%)$.

\section{Os presos baianos e os transtornos psiquiátricos}

A prevalência de transtorno psiquiátrico entre prisioneiros no Brasil e na Bahia é pouco conhecida e estudada. Os dados encontrados no presente estudo confirmam resultados de estudos realizados em outros países apontando para prevalências mais altas de transtornos psiquiátricos entre presos do que na população geral. Não houve diferença significativa na prevalência de depressão entre os dois regimes prisionais. A prevalência de depressão ao longo da vida foi de 12,8 a $16,9 \%$, estimativa inferior aos 22,9\% em população carcerária na França. (FALISSARD et al., 2003) Com relação a episódio depressivo atual a prevalência foi de 5,2 a 7.2\%, resultado também inferior à média de 10\% estimada em estudo metanalítico. (FAZEL; DANESH, 2002) 
Com relação a transtornos mentais mais graves, especificamente transtorno bipolar do humor e transtorno psicótico, as prevalências foram significativamente maiores para os presos em regime semiaberto. Esse dado provavelmente reflete o fato da falha da assistência psiquiátrica no regime fechado, levando, provavelmente, à transferência para o hospital de custódia dos presos com transtorno mentais graves nesse regime. Comparando com os dados da literatura mundial a prevalência de psicose do regime semiaberto foi de 12,6\%, semelhante aos 13,4\% encontrados na França (FALISSARD et al., 2006) e 11,5\% nos Estados Unidos (GUY et al., 1985) e maior do que aquela estimada em estudo metanalítico que foi de 3 a 7\%. (FAZEL; DANESH, 2002) A prevalência de psicose no regime fechado foi semelhante à prevalência geral na população, 1,4\%, e menor do que aquela relatada na literatura.

Com relação ao transtorno antissocial de personalidade as prevalências foram semelhantes entre os dois regimes prisionais $(24,2 \%$ a $26,9 \%$ ) sendo menor do que os $47 \%$ referidos em estudo metanalítico. (FAZEL; DANESH, 2002) Com relação ao trantorno borderline de personalidade a prevalência foi significativamente maior entre presos do regime semi-aberto (34,8\%) em relação ao regime fechado (19,7\%), sendo relativamente semelhante aos $25 \%$ reportados em estudo metanalítico. (FAZEL; DANESH, 2002)

A prevalência de transtornos de ansiedade não diferiu significativamente entre os dois regimes prisionais. A prevalência de transtorno de ansiedade generalizada (4,1 a 7,2\%) também foi menor do que os 15,4\% reportados em prisões na França FALISSARD et al., 2006). A dependência e o abuso atual de álcool foram significativamente mais frequentes entre os presos em regime semiaberto. Como o regime semiaberto possibilita um maior contato com o mundo externo, esse pode ser um fator relacionado à maior prevalência do consumo abusivo e dependência atual entre os presos do regime semiaberto. $\mathrm{O}$ abuso durante a vida não difere entre os dois regimes, porém a dependência durante a vida foi significativamente maior para os presos do regime semiaberto, o que nos leva a pensar que talvez existam características comuns entre os presos em regime semiaberto em relação àqueles em regime fechado que os levem a um maior risco de dependência de álcool. No que concerne a problemas relacionados ao uso de outras substâncias a dependência atual de substâncias foi significativamente 
maior entre os presos em regime semiaberto. $\mathrm{O}$ abuso atual e a dependência durante a vida, contudo, não diferiu entre os dois regimes.

Em comparação com outros estudos, o presente estudo indica prevalências mais elevadas de problemas relacionados ao uso de substâncias. Falissar et al. (2006) estimaram uma prevalência de 8,7\% de dependência de álcool, atual ou durante a vida e 8,9\% de dependência de droga, atual ou durante a vida. (FALISSARD et al., 2006) Guy et al. (1985) estimaram em 25\% uso ou dependência de álcool e 11,5\% de drogas. (GUY et al., 1985) No presente estudo, a dependência ou o abuso atual ou durante a vida chegam a 35\% para álcool e 32,4\% para outras substâncias. Como as entrevistas foram realizadas no pátio do presídio e não em área administrativa, pode-se pensar que os internos tenham se sentido mais à vontade para expressar atos que poderiam ser considerados ilícitos. Esses dados indicam um grave problema relacionado ao abuso e dependência de substâncias entre criminosos no Estado da Bahia, Brasil.

A prevalência de TDAH na vida adulta foi semelhante entre os dois regimes prisionais $(4,1 \%$ no regime fechado e 5,3\% no regime semiaberto) e à estimativa de $4,4 \%$ relatada na literatura recente. (KESSLER et al., 2006) As prevalências de TDAH na infância foram significativamente maiores entre os presos em regime semi-aberto, enquanto que a prevalência entre adultos não diferiu entre os dois regimes. Considerando que nem sempre o adulto se lembra com exatidão a época em que teve determinados sintomas, apresentamos os dados considerando que os sintomas apareceram na infância sem considerar a idade de início. No regime fechado a prevalência de 10,3\% se aproxima àquela da literatura para crianças escolares na mesma região, que estimou uma prevalência de 6,7\%. (PONDÉ; FREIRE; 2007; FREIRE; PONDÉ, 2005) No que concerne ao regime semiaberto, contudo, as estimativas de $22,2 \%$ são bastante superiores àquelas encontradas na literatura de referência. Estudos de seguimento mostram que existe uma elevada tendência à criminalidade e atos anti-sociais entre adolescentes e adultos que tiveram TDAH na infância. (MANUZZA et al., 1989; MANUZZA et al., 2008) Pode-se supor que os presos em regime semiaberto tenham cometido crimes menos graves do que aqueles em regime fechado e que assim as crianças com TDAH na infância talvez estejam propensas à criminalidade, porém com crimes menos 
graves na vida adulta. Esses dados devem ser investigados em estudos posteriores que analisem os tipos de crimes cometidos entre aqueles que tiveram TDAH na infância e aqueles que não foram acometidos por essa doença.

\section{Referências}

FAZEL, S; DANESH, J. Serious mental disorder in 23000 prisoners:

a systematic review of 62 surveys. Lancet, v. 359, n. 9306, p. 545-50, 2002.

BRUGHA, T. et al. Psychosis in the community and in prisons: a report from the British National Survey of psychiatric morbidity. Am J Psychiatry, v. 162, n. 4, p. 774-80, 2005.

FALISSARD, B. et al. Prevalence of mental disorders in French prisons for men. BMC Psychiatry, v. 21, p. 6-33, 2006.

MORRIS, M. Managing the unmanageable: psychotherapy in Grendon Prison. Crim Behav Ment Health, v. 12, n. 2, p. 54-67, 2002.

WEXLER, HK. The promise of prison-based treatment for dually diagnosed inmates. J Subst Abuse Treat,v. 25, n. 3, p. 223-231, 2003

SMITH, S. S; BAXTER. V. J; HUMPHREYS, M. S.Psychiatric treatment in prison: a missed opportunity? Med Sci Law, v. 43, n. 2, p. 122-126. 2003.

BIRMINGHAM, L; MASON, D; GRUBIN, D. A follow-up study of mentally disordered men remanded to prison. Crim Behav Ment Health, v. 8, p. 202-213. 1998

LANGEVELD, H; MELHUS, H. Are psychiatric disorders identified and treated by in-prison health services? Tidsskr Nor Laegeforen. v. 124, n. 16, p. 2094-2097, 2004.

BUTLER, T. et al. Mental disorders in Australian prisoners: a comparison with a community sample. Aust N Z J Psychiatry, v. 40, n. 3, p. 72-6, 2006. BROOKE, D. et al;. Point prevalence of mental disorder in unconvicted male prisoners in England and Wales. BMJ. v. 313, n. 7071, p. 1524-7, 1996.

ASSADI, S. M. et al. Psychiatric morbidity among sentenced prisoners: prevalence study in Iran. Br J Psychiatry. v. 188, p. 159-64, 2006.

AMORIM, P. Mini International Neuropsychiatric Interview (MINI): validação de entrevista breve para diagnóstico de transtornos mentais. Rev Bras Psiquiatr.v. 22, n. 3, p. 106-15, 2000. 
GUY, E; PLATT J. J; ZWERLING, I.; BULLOCK, S. Mental health status of prisoners in an urban jail. Crim Justice Behav. v. 12, n. 1, p. 29-53, 1985.

KESSLER, R. C. et al. The prevalence and correlates of adult ADHD in the United States: results from the National Comorbidity Survey Replication. Am J Psychiatry. v. 163 n. 4, p. 716-23, 2006.

PONDÉ, M. P; FREIRE, A. C. Prevalence of attention deficit hyperactivity disorder in schoolchildren in the city of Salvador, Bahia, Brazil.

ArqNeuropsiquiatr, v. 65, p. 240-244, 2007.

FREIRE, A. C; PONDÉ, M. P. A pilot study of prevalence of hyperactivity and attention deficit disorder in scholar children in Salvador, Bahia, Brazil. Arq Neuropsiquiatr, v. 63, n. 2B, p. 474-8, 2005.

MANNUZZA, S. et al. Hyperactive boys almost grown up. IV. Criminality and its relationship to psychiatric status. ArchGen Psychiatry, v. 46, n. 12, p. 1073-9, 1989.

MANNUZZA, S; KLEIN, R. G, MOULTON, J. L. Lifetime criminality among boys with attention deficit hyperactivity disorder: a prospective follow-up study into adulthood using official arrest records. Psychiatry Res, v. 160, n. 3, p. 237-46, 2008. 